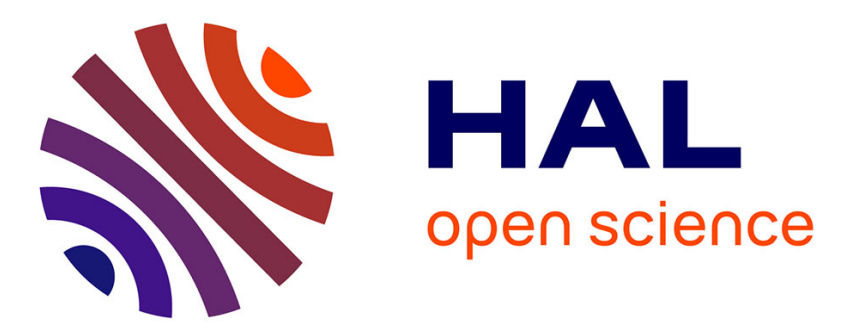

\title{
De Smyrne à Philae: contacts et rencontres de P. Ælius Aristide en Égypte
}

Constantin Raïos

\section{To cite this version:}

Constantin Raïos. De Smyrne à Philae: contacts et rencontres de P. Ælius Aristide en Égypte. Archimède: archéologie et histoire ancienne, 2019, La République "gréco-romaine " des lettres: construction des réseaux savants et circulation des savoirs dans l'Empire romain, HS Nº1, pp.68 à 81 . halshs-02091587

\section{HAL Id: halshs-02091587 https://shs.hal.science/halshs-02091587}

Submitted on 5 Apr 2019

HAL is a multi-disciplinary open access archive for the deposit and dissemination of scientific research documents, whether they are published or not. The documents may come from teaching and research institutions in France or abroad, or from public or private research centers.
L'archive ouverte pluridisciplinaire HAL, est destinée au dépôt et à la diffusion de documents scientifiques de niveau recherche, publiés ou non, émanant des établissements d'enseignement et de recherche français ou étrangers, des laboratoires publics ou privés. 


\title{
DE SMYRNE À PHILAE : CONTACTS ET RENCONTRES DE P. AELIUS ARISTIDE EN ÉGYPTE *
}

\author{
Constantin RAIIOS \\ Maître de conférences en Langue et \\ littérature grecques anciennes et modernes \\ Université Toulouse - Jean Jaurès \\ EA 4601 PLH-CRATA \\ constantinos.raios@univ-tlse2.fr
}

\section{RÉSUMÉ} * Le point de départ du présent article repose sur des suggestions
de deux membres de notre jury de thèse, M. le Prof. P. Goukowsky,
Membre de I'Institut, et M. le Prof. P. Heilporn, Directeur de I'Institut
de Papyrologie de I'Université de Strasbourg. Qu'ils reçoivent nos sin-
cères remerciements. Nous tenons à remercier aussi nos collègues de
I'Université Toulouse - Jean Jaurès, J.-C. Carrière (Professeur émérite)
et O. Spevak (Maître de conférences) pour leurs remarques et conseils.

Presque l'ensemble de l'œuvre d'Ælius Aristide évoque constamment l'entourage (parents, collègues, élèves, gouverneurs, admirateurs, médecins, prêtres) et les déplacements du sophiste. Dans le présent article, nous nous proposons d'examiner les rapports existant entre une partie du réseau d'Aristide et un de ses voyages de jeunesse. En confrontant des sources littéraires à des inscriptions et à des données prosopographiques,

\section{MOTS-CLÉS}

Ælius Aristide, Égypte gréco-romaine, Nil,

réseau,

voyages,

exil,

honneur,

rhétorique,

Asclépios,

administration. nous illustrons le rôle joué par quelques connaissances d'Aristide avant, pendant et après son séjour en Égypte. Enfin, nous étudions les diverses façons dont l'auteur a su profiter de ses rencontres dans les domaines social, religieux et rhétorique.
Almost all Ælius Aristides' works constantly evoke his entourage (parents, colleagues, disciples, governors, admirers, medicines, priests) and his journeys. In the present article, I first examine the existing relationships between a segment of Aristides' network and one of his youth travels. By bringing together literary sources, inscriptions and prosopographic data, I clarify the role of some acquaintances of the sophist before, after and during his stay in Egypt. Finally, I study the various ways that the author succeeds in making good use of his contacts in a social, religious and rhetoric field.
KEYWORDS

Ælius Aristide, Greek and Roman Egypt, Nile,
network, travels, exile, honor, rhetoric, Asclepios, administration. 
Les représentants de la Seconde Sophistique, venant des quatre coins de l'Empire romain, enseignaient et pratiquaient l'art rhétorique devant un public qui, quelles que fussent ses origines, pouvait se permettre le prix et le temps de cet apprentissage. Dotés souvent de la citoyenneté romaine, les orateurs pouvaient être sollicités par leur patrie, leur cité d'adoption ou une autre collectivité qui aurait besoin de leur éloquence pour demander quelque privilège auprès des autorités, pour défendre sa position lors d'une dispute, pour accueillir un dignitaire du pouvoir central ou pour animer des cérémonies d'ordre civique et religieux. [1] Les sophistes sont progressivement devenus les intermédiaires, voire les acteurs, principaux de l'osmose des cultures grecque et romaine. Dans leurs rangs on trouve des occidentaux hellénisés, comme Favorinos d'Arles, des Grecs issus de l'espace de l'Hellade classique, comme Hérode Atticus, ou originaires de I'Asie Mineure, tel que Polémon de Laodicée, et des orientaux, comme Lucien de Samosate. Tous, tantôt pour compléter leur formation, tantôt pour faire croître leur réputation et le nombre de leurs disciples, sillonnaient les provinces romaines, se liaient d'amitié avec leurs élèves et rencontraient leurs collègues ou d'autres intellectuels. Parmi ces derniers, on trouve des grammairiens, des philosophes, des poètes, des prêtres de cultes de toutes sortes et des représentants du

[1] Outre les synthèses de BoulANGER 1923, de BOWERSOCK 1969 et de WHITMARSH 2005, voir HOSTEIN \& LALANNE 2012

[2] PERNOT 2007, p. 938-939.

[3] Sur sa vie et son œuvre, voir à titre indicatif BOULANGER 1923 ; WILAMOWITZ-MOELLENDORFF 1925 ; BEHR 1994 ; PERNOT 1989.

[4] Sur la datation du voyage à Rome et du discours qui y fut prononcé, voir PERNOT 1997, p. 163-170. pouvoir romain, dont les fonctions pouvaient varier de celles de simple magistrat local à celles de sénateur ou même d'empereur.

$\mathrm{Si}$, par le présent article, nous nous proposons d'étudier une partie du réseau d'Ællius Aristide, ce n'est pas uniquement du fait qu'il s'agit d'une figure importante de la Seconde Sophistique, mais surtout à cause de la «préoccupation autobio-bibliographique constante » qui caractérise son œuvre, car l'orateur essaie d'immortaliser par chacun de ses discours publiés les hauts lieux de sa carrière et de son évolution spirituelle. [2] Par conséquent, l'ensemble de sa production littéraire s'avère d'une inestimable utilité pour mieux s'apercevoir de la vie privée ou publique des sophistes et du tissage de leurs réseaux intellectuels.

Publius Aelius Aristide ( $\Pi$ ó $\pi \lambda ı$ Ios Aï $\lambda_{\text {Ios }}$ 'ApıotzíOns) [3] est né en 117 ap. J.-C. à Hadrianoi, une petite ville en Mysie, au nord de l'Asie Mineure, et ses tria nomina témoignent que ses parents avaient acquis la citoyenneté romaine sous la dynastie des Antonins. Élevé dans un environnement aisé et cultivé, il a reçu une éducation soignée : adolescent, il a quitté le foyer familial pour parfaire son éducation littéraire à Smyrne auprès du grammairien Alexandros de Kotiaion, futur maître de Marc Aurèle, et pour suivre les cours des grands orateurs de son temps, comme Polémon de Laodicée.

Vers la fin de son éducation rhétorique et avant de se lancer dans la carrière sophistique, il a réalisé un voyage en Égypte (après 137 et avant 143). En remontant le cours du Nil, il a été confronté à des problèmes de santé (Or., 36, § 49), sans doute pour la première fois de sa vie, mais c'est durant et après son premier voyage à Rome en 144 [4] que son état s'est aggravé et l'a amené au sanctuaire d'Asclépios à Pergame pour lutter contre une maladie qu'il considérait comme 
incurable par la médecine rationnelle. Depuis lors, la vie et l'art rhétorique d'Aristide ont été rendus indissociables du culte de la divinité qui le guidait par des ordonnances et des thérapies, souvent à l'encontre des conseils des médecins. Les cinq Discours sacrés (Or., 47-52) relatent à la première personne une période de presque vingt-trois ans passée sous le patronage d'Asclépios et fournissent des informations de première main sur les activités de l'orateur.

L'utilité du corpus n'a échappé ni aux biographes d'Aristide ni à Emma et Ludwig Edelstein qui, dans leur ouvrage classique sur le culte d'Asclépios [5], ont tiré profit des Or., 47-52 pour décrire le monde des temples du dieu thérapeute. Enfin, au cours des dernières décennies, deux articles de $\mathrm{H}$. Remus et de L. Quattrocelli [6] ont abordé la question du public et des amis d'Aristide à travers le même matériel littéraire. [7]

En annonçant ce que L. Pernot interpréterait quelques années plus tard comme la transformation du triangle hippocratique « malade - maladie - médecin » en un triangle formé par Aristide, ses discours et Asclépios [8], Remus a signalé la corrélation établie entre la santé fragile d'Aristide, ses amis, sa foi et ses succès professionnels. Les hommes que fréquentait Aristide dans les sanctuaires ne consti-

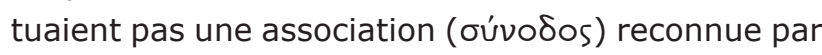
les autorités, mais ils se rapprochaient simplement les uns des autres par le biais de leurs intérêts communs. Remus a aussi tenté de dégager les degrés de l'ami-

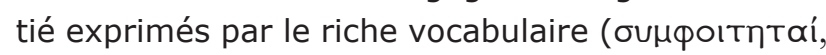

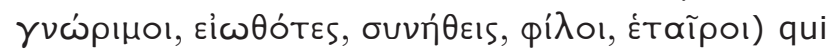
décrit les relations d'Aristide dans ses discours. Quant à Quattrocelli, qui a travaillé indépendamment de Remus, elle a étudié les conditions de présentation, le style et les buts de chacun des Discours sacrés pour distinguer finalement un cercle d'amis restreint

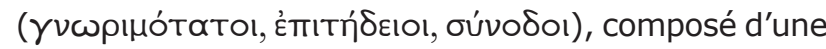
cinquantaine de résidents smyrniotes, d'avec un groupe hétérogène de nombreux adeptes du culte d'Asclépios qui assistaient aux diverses manifestations de dévotion d'Aristide.

\section{[5] EDELSTEIN \& EDELSTEIN 1945}

[6] Remus 1996 ; QUATTROCELLI 2009.

[7] Un autre corpus de textes aristidiens susceptible de fournir de précieux renseignements sur l'entourage de l'orateur mysien est constitué par les Or., 30-34, qui portent sur la formation et les activités pédagogiques d'Aristide ; voir VIX 2010

[8] PERNOT 2002, p. 371.

[9] Comme sa tpopós, Philoumène (Or., 47, § 78), et son трофвú, Z Zosimos (Or., 47, § 69-77).

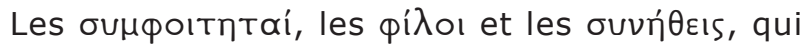
côtoyaient le sophiste à I'intérieur ou à l'extérieur des sanctuaires et des thermes d'Asie Mineure, comptaient dans leurs rangs, hormis les membres de sa famille [9], des hommes politiques, des prêtres, des médecins et des simples fidèles ( $\theta \varepsilon \rho \alpha \pi \varepsilon u \tau \alpha$ í) avec des intérêts intellectuels [10]. Bien que les renseignements prosopographiques fournis par les textes relatifs à la période de la vie d'Aristide avant sa maladie soient moins détaillés, ils décrivent de manière satisfaisante le contour du monde dans lequel circulait le sophiste et, une fois mis en parallèle avec les Or., 47-52 et quelques témoignages épigraphiques, ils permettent de faire ressortir les traits généraux de ses priorités, de son public et de ses relations avec le pouvoir.

\section{EN АIГYПTWI ET ПEPI АIГYПTOY}

Le voyage et le séjour d'Aristide en Égypte I'ont marqué à tel point que même ses rêves étaient envahis par des paysages égyptiens. Au § 24 de l'Or. 47, le sanctuaire d'Apollon de Milyas, en Asie Mineure, est confondu avec celui d'Isis à Philae ; [11] aussi, à I'Or. 49 (§ 3-4), Aristide se voit-il naufragé au large du littoral égyptien : après son sauvetage, il se croit dans les rues d'Alexandrie, où il entend, à sa grande satisfaction, la récitation d'un de ses poèmes par les élèves d'une école de la ville.

Le « souci autobio-bibliographique »d'Aristide ne pouvait accepter qu'un discours inspiré de cette terre fabuleuse soit absent de la liste des titres de sa production littéraire. Après son retour et son entrée dans le cercle des dévots d'Asclépios, [12] il a rédigé le Discours égyptien (Or., 36), le seul à relater cette étape de sa vie. Une autre singularité dudit discours est qu'il traite d'une question naturelle, le problème des crues du Nil. Toutefois, le véritable objectif de I'Or. 36 n'est ni de résoudre l'énigme scientifique ni de corroborer une explication déjà fournie par la très riche tradition ancienne. [13] Le sophiste, s'adonnant

[10] Tels que Pardalas (Or., 50, § 27), L. Sedatius Theophilus de Nicée (Or., 48, § 18 et Or., 50, § 16-17) et Q. Tullius Maximus (Or., 50, § 18).

[11] La visite d'Aristide à Éléphantine et à Philae est décrite aux $\S 46-50$ de l'Or. 36 , bien que le sanctuaire ne soit pas explicitement mentionné. À propos de la superposition des deux lieux, voir FOURNEL 2015, p. 1-3.

[12] Aristide mentionne les cures qu'Asclépios lui suggère au § 124 de I'Or. 36.

[13] Sur cette énigme, voir BONNEAU 1964 ; POSTL 1970 ; MANOLARAKI 2012. 
plutôt à un exercice rhétorique, [14] attribue le phénomène à l'origine et à la nature divines du fleuve, face auxquelles la science humaine reste désemparée. Aristide réfute les principaux motifs de la crue proposés par ses devanciers [15] pour aboutir à une célébration de la divinité du fleuve en récapitulant I'ensemble des paradoxes liés au Nil.

Grâce à I'Or. 36, on est en mesure de confirmer que le point de base d'Aristide était situé au nord du pays : outre la mention d'Alexandrie aux § 33-34, les termes employés pour désigner les excursions en

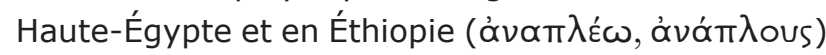
montrent clairement que le sophiste devait remonter le cours du Nil. Le premier paragraphe de I'Or. 36 énumère les monuments visités sur les traces d'Hérodote : les pyramides, les grands temples et le labyrinthe de Crocodilopolis. Aristide a effectué des voyages aux environs de Thèbes ( $\S 33$ ), à Canope ( $\S 109$ ), célèbre lieu touristique et religieux de son temps, [16] dans le nome d'Hermonthis (§ 33), à Philae, à Syène et à Éléphantine (§ 47-54) ; enfin, il a dû traverser une partie du désert arabique en Égypte orientale ( $\$ 30$ ) et le territoire de Pselchis (§ 81), [17] avant d'atteindre la limite méridionale de l'Empire romain, la frontière éthiopienne ( $§ 31$, $48,55)$. [18] Pourtant, la perte réelle ou prétextée des notes prises pendant son séjour en Égypte ( $§ 1$ :

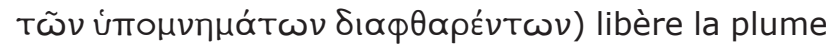
de l'auteur des entraves d'un récit exclusivement oculaire et lui permet de se tourner vers diverses sources écrites pour s'engager dans un dialogue avec les auteurs du passé. [19]

Suivant la tradition qui considérait les prêtres égyptiens comme les gardiens séculaires de la sagesse humaine, il commence sa propre enquête par leur rencontre. Dans I'exorde, Aristide se souvient des conversations avec eux :

[14] Selon les Progymnasmata d'Aelius Théon (Patillon 2 = Spengel 66-67), l'étude des arguments d'Éphore contre les anciennes théories portant sur la crue du Nil était conseillée aux apprentis rhéteurs.

[15] Seuls les auteurs littéraires, poètes et historiens, sont explicitement cités par l'orateur, tandis que les noms des philosophes sont passés sous silence (BEHR 1981, p. 403).

[16] Cf. Strabon, XVII, 1, 17.

[17] FOERTMEYER 1989, p. 183.

[18] Sur cette étape du séjour égyptien d'Aristide, voir ROBIANO 2011.

[19] REARDON 1971, p. 224 semble convaincu que les notes prises pendant le voyage en Égypte étaient destinées à la rédaction d'un exposé périégétique.

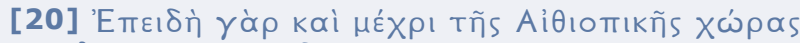

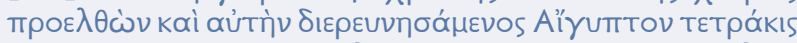

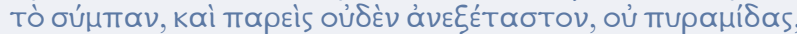

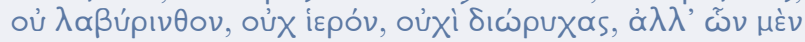

1. [20] En effet, je me suis avancé jusqu'au pays d'Éthiopie, j'ai exploré l'Égypte elle-même quatre fois au total et je n'ai rien laissé sans l'examiner, ni pyramides ni labyrinthe ni sanctuaires ni canaux ; au contraire, quand les mesures des monuments se trouvaient dans les livres, je me les suis procurées là, et quand elles n'étaient pas disponibles, j'ai pris les mesures moi-même avec les prêtres et les prophètes [21].

La sagesse des ministres des dieux égyptiens n'était pas seulement anecdotique, [22] puisque certains parmi eux, comme par exemple les scribes, étaient de vrais spécialistes dans le domaine de la topographie des lieux saints et des monuments. Qui plus est, Aristide, à l'instar d'Hérodote, [23] recourt souvent dans ses hymnes en prose à la primauté des Égyptiens en matière de théologie. Pour défendre le caractère guerrier d'Athéna (Or., 37, § 14), le sophiste évoque l'invention du bouclier par les favoris de la déesse, les Athéniens en Europe et les Égyptiens en Asie ; quant à la nature divine d'Héraclès, elle est indiscutable, car le fils de Zeus fait partie des panthéons égyptiens et phéniciens (Or. , 40, § 10) ; enfin, I'importance du culte de Sarapis en Égypte certifie la grande puissance du dieu (Or., 45, § 15). [24]

D'une manière analogue, Aristide utilise dans I'Or. 36 les témoignages du clergé local pour consolider son argumentation contre les idées reçues des Grecs sur l'Égypte et le Nil. Vers la fin du discours (§ 104-113), Aristide concentre ses tirs sur les récits relatifs au voyage de Ménélas : selon Hécatée de Milet, [25] la ville de Canope serait nommée ainsi pour commémorer la mort du pilote grec Canopos, mais le sophiste, à l'instar d'Hérodote (II, 15 et 77) et de Platon (Timée, 22c-e), se fie à l'autorité d'un prêtre, qui lui enseigne I'origine égyptienne du toponyme et écarte les interprétations grecques. [26]

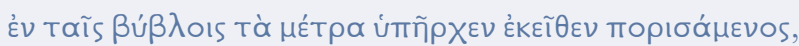

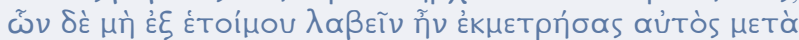

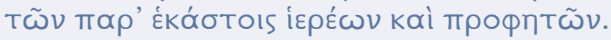

[21] Par le terme профп்тп,, Aristide désigne les prêtres égyptiens de haut rang. Cf. Apulée, Met., 2, 28: propheta primarius.

[22] Sur les connaissances légendaires et réelles du clergé égyptien dans la tradition grecque, voir SAUNERON 1988, p. $119-128$.

[23] II, 50-59.

[24] Voir le commentaire ad loc. dans GoEKEN 2012.

[25] FrHGr I 1, fr. 307-309.

[26] Or., 36, 108-109. Notons que pour expliquer les noms propres, les prêtres égyptiens avaient I'habitude de faire appel à des étymologies populaires, souvent fondées sur une ressemblance superficielle des mots (voir SAUNERON 1988, p. 133-136). 
De surcroît, même les profanes étaient censés être mieux placés pour parler de la nature de leur propre pays que les auteurs grecs du passé. En réfutant la possibilité de l'existence de neige en Égypte et en Éthiopie, dont la fonte était considérée comme le motif de la crue du Nil par Euripide et Eschyle, [27] Aristide rapporte avec étonnement (Or., 36, § 15) qu'en dépit des efforts de ses interprètes, il n'a pas été en mesure de décrire ce phénomène météorologique aux Égyptiens qui ne parlaient pas grec (ö $\lambda \omega s$ $\beta \alpha \rho \beta \alpha \rho i \zeta O v T \varepsilon s)$ et qui n'avaient jamais voyagé à l'étranger. De même, comme les Éthiopiens vivent plus près des sources du Nil que tout autre peuple, ils sont des témoins dignes de foi pour parler de ce qui se passe dans les contrées du Sud peu ou mal explorées. Ainsi, l'auteur se tourne vers eux pour corroborer sa réfutation de la théorie de Démocrite et du Péripatos ( $\S 31$ ), puis pour démentir celle d'Hérodote (§ 55-57). [28]

En général, I'orateur traite les autochtones avec respect, voire avec admiration, mais ses contacts avec eux ne sortent pas des limites du voyage culturel et n'aboutissent pas à des échanges sur de longs termes. Aristide ne les lie pas à ses autres activités ou à son cercle d'amis : ces hommes déconstruisent par leurs témoignages les préjugés sur l'histoire et la géographie de l'Égypte ou de l'Éthiopie, bien que finalement, en confirmant leur sagesse proverbiale, ils agissent de manière conforme aux attentes des Grecs. Les rencontres en question sont, donc, destinées à faire partie des souvenirs de voyages d'Aristide et à alimenter les discussions du véritable réseau de l'orateur.

Au fait, la thématique de l'Or. 36 devait répondre aux intérêts encyclopédiques des fréquentations du sophiste en Asie Mineure. Signalons que les premiers paragraphes du discours ont une forme épistolaire, sans que le nom du destinataire ne soit précisé. La rédaction des missives était courante dans le domaine des sciences exactes et permettait aux auteurs de faire part d'une découverte ou de répondre rapidement à une question posée par leurs amis, [29] comme Aristide se propose de faire dans les lignes qui ouvrent le discours. [30] De plus, le style ordinairement flexible de l'épistolographie grecque laisse un atticiste utiliser un vocabulaire technique, contemporain ou d'origine étrangère, [31] sans pour autant faire trop de concessions ou donner prise aux critiques. [32]

Le lecteur de I'Or. 36 repère au moins trois allusions à des connaissances de l'auteur qui partageaient les mêmes préoccupations intellectuelles et religieuses que lui. Le premier s'appelait Dion :

10. [33] Quant à moi, en tout cas, je l'ai appris d'un homme qui, pour citer Démosthène, n'est aucunement capable de mentir : notre compagnon Dion ( $\Delta$ íwvos ÉTaípov), qui est expérimenté en matière de faits et de paroles. Celui-ci me raconta qu'en été il voyageait sur un navire vers I'Égypte et que la terre n'était pas encore visible ; au contraire, elle se trouvait si loin qu'il était même impossible de conjecturer la distance. Les marins, soit par besoin soit parce qu'ils voulaient simplement faire une démonstration aux passagers, puisèrent une eau pure et potable d'en bas à une grande distance.

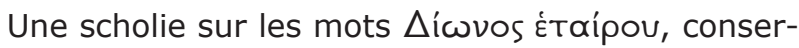
vée dans le plus ancien manuscrit de I'Or. 36, le Florentinus Laurentianus plut. LX, 3 (f. 142v), et composée en deux phases, identifie initialement l'homme avec Dion de Pruse, dit Chrysostome, avant de rejeter I'hypothèse pour des raisons chronologiques [34]. Bien que le verbe ớkoúc décrive parfois le processus de la
[27] Il s'agit en réalité de l'explication d'Anaxagore : voir BONNEAU 1964, p. 161-169.

[28] Le débat sur la présence d'un notable éthiopien en territoire romain est résumé et commenté par RoBIANO 2011 , p. $145-146$.

[29] SYKUTRIS 1931.

[30] Aristide écrivait des lettres et des discours sous l'impulsion du moment et à la suite des événements concrets (Or., 17, 18, 19, 20, 22, 23 et 24), mais le destinataire de I'Or. 36 pourrait être un personnage fictif. Voir les remarques de ROBERT 2013, p. 1-2, sur la disparition progressive de la deuxième personne dans I'Or. 36.

[31] Voir, à titre indicatif, l'emploi des mots et des expres-

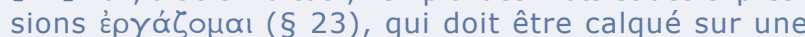

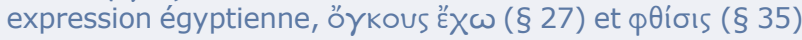
dans I'Or. 36.

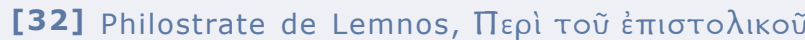

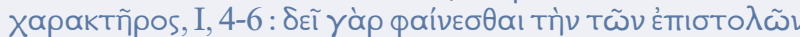

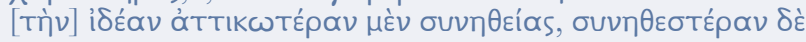

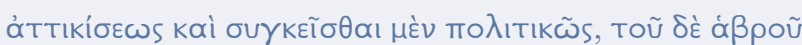

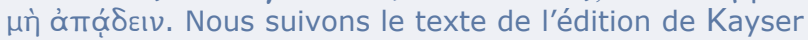
1870-1871 avec les corrections proposées par RAÏOs 1992, p. 162-165 ; entre les pages 169 et 175 du même ouvrage, il est aussi possible de consulter les editiones principes des deux traductions manuscrites du texte grec en latin et en français.

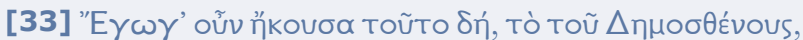

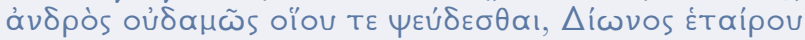

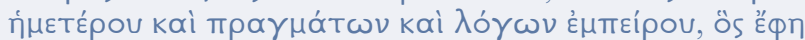

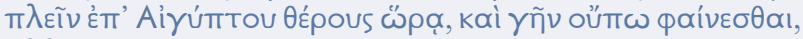

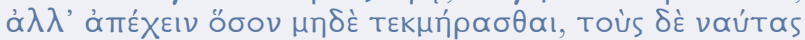

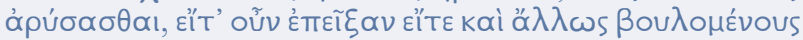

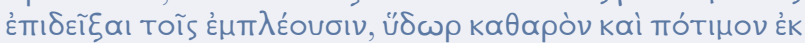

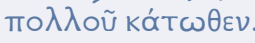

[34] Une édition préliminaire de la scholie peut pour I'instant être consultée dans notre thèse de doctorat : RAÏOS 2011. Nous espérons qu'une nouvelle édition critique de I'Or. 36 et de ses scholies anciennes ou médiévales paraîtra bientôt dans la CUF. 
lecture [35], nous pouvons écarter la possibilité de la citation d'un collègue défunt, car Aristide préfère quali-

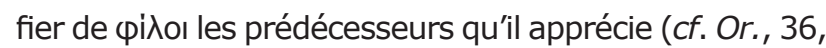
$\S 57)$ et réserver દ̇ंaĩpos pour ses contemporains [36].

Behr [37], en partant du fait que par Étaĩpos l'orateur s'adresse souvent à ses amis de I'Asclépieion de Pergame, émet arbitrairement I'hypothèse que Dion proviendrait du même milieu. Au contraire, Remus [38] note justement que ÉTaĩpos est aussi utilisé par Aristide quand il parle des collègues ou d'autres proches, tels que I'orateur Quadratus (Or., 50,63 ) ou son maître, Alexandros de Cotiaion (Or., $32,2)$, sans que ces hommes ne soient nécessairement associés au culte d'Asclépios. Par conséquent, le savant canadien classe Dion dans la catégorie des « professional companions-friends ». D'ailleurs, l'ex-

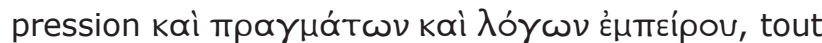
en affermissant la véracité des souvenirs rapportés, esquisse le portrait-type d'un sophiste qui effectue des séjours dans des provinces éloignées pour compléter sa formation ou pour enrichir son parcours. On voit, donc, qu'outre I'homonymie avec Dion de Pruse, c'est la description sommaire du personnage qui a dû jouer son rôle dans la composition de la scholie initiale du manuscrit florentin.

Plus loin, dans une séquence de l'Or. 36 consacrée à la réfutation de la théorie d'Éphore, [39] un ouïdire est encore cité ( $\$ 82$ ) à propos d'un lac près de Scythopolis. Nous ignorons les conditions dans lesquelles Aristide a rencontré ses hôtes ( $\xi \dot{v} v o ı), ~[40]$ mais la réminiscence des discussions portant sur des paradoxes naturels est encore une fois palpable. Enfin, Aristide, vers la fin de I'Or. 36 (§ 125), renvoie à

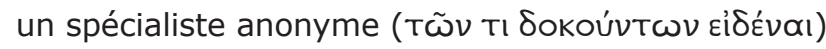
du culte égyptien. De nouveau, l'auteur ne donne pas beaucoup de précisions sur son ami, mais il est possible que le connaisseur soit un autre adepte des divinités égyptiennes à Smyrne, voire un prêtre ; la prudence avec laquelle le sophiste le laisse parler à sa place des pratiques concernant le culte du Nil n'est pas sans rappeler le $\S 15$ de I'Or. 45, où Aristide

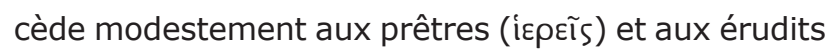
( $\lambda$ óyıı ) égyptiens le soin d'aborder la nature divine du dieu sauveur.

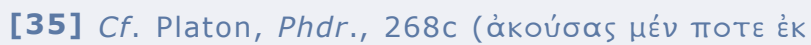
$\beta$ ı $\beta$ íou ) ; Plutarque, Sur les délais de la justice divine, 2

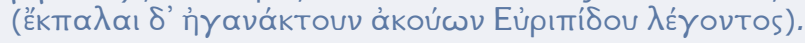
[36] Le § 24 de I'Or. 50 est révélateur des habitudes d'Aristide, car les auteurs des siècles précédents (a̋ $v \delta \rho \varepsilon s$

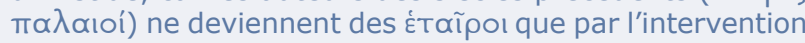
miraculeuse d'Asclépios.

[37] BEHR 1968, p. 17, n. 53
Si la présence du sanctuaire de Philae dans les rêves du sophiste (Or., 47, 24) permet de sous-entendre qu'il l'a vu de ses yeux, aucune visite de temple n'est relatée dans I'Or. 36. Toutes les discussions rapportées ou résumées avec les prêtres d'Égypte ne concernent que I'histoire et la géographie du pays, tandis que les questions spirituelles ou théologiques brillent par leur absence et s'opposent, à première vue, à l'implication bien attestée d'Aristide dans le culte des dieux égyptiens. Ce paradoxe, qui contraste avec le véritable enjeu de I'Or. 36, est justifié par la démarche rhétorique et scientifique du discours. En effet, l'importance que le sophiste accordait à Sarapis ne devrait pas être cantonnée à son séjour en Égypte ; peut-être faut-il voir derrière la discrétion d'Aristide une manifestation de piété et $d$ 'humilité face à ses vénérables initiateurs.

Il est vrai que dans les cas précédents, on reste sur sa faim, faute de renseignements précis sur les individus que cite Aristide, mais il ne serait pas trop hasardeux de suggérer que tous ces personnages devaient appartenir aux cercles d'érudits et/ou de dévots des dieux thérapeutes gréco-égyptiens que I'orateur fréquentait en Asie Mineure. Néanmoins, même si Dion, les そ̇ंvo (de Scythopolis ?), le théologien anonyme, les Égyptiens et les Éthiopiens permettent au lecteur de se faire une idée de la nature des discussions savantes fondées sur les souvenirs de voyages, ils ne donnent aucun indice sur les autres activités d'Aristide en Égypte.

\section{RETROUVAILLES, CONTACTS ET SOUVENIRS : LE RÉSEAU ÉGYPTIEN APRĖS LA FIN DU SÉJOUR EN ÉGYPTE}

Hormis les éléments égyptiens présents dans les rêves des Or. 47-52, deux personnes que le sophiste a dû rencontrer lors de son séjour dans le pays des Pharaons sont évoquées dans les pages du quatrième Discours sacré (Or., 50), à savoir : un philosophe grec et un haut fonctionnaire romain. Une partie de I'Or. 50 (§ 14-21) traite des miracles et des visions qui ont persuadé Aristide de se livrer aux soins d'Asclépios ainsi que de ne pas abandonner la rhétorique à cause

[38] REMUS 1996, p. 150-151, 168 et 171 (n. 21).

[39] BONNEAU 1964, p. 184-186 ; PARMEGgIANI 2011, p. $68-70$.

[40] L'extrait, accompagné des $\S 88$ et 89 , est inclus dans le corpus de STERN 1980, n³71, p. 218-219. Stern, en forçant légèrement le texte grec, s'est permis d'en déduire qu'Aristide a visité Scythopolis. 
de ses problèmes de santé au cours de l'année 145. Des rêves, par lesquels le dieu sauveur invitait divers personnages à soutenir l'orateur en difficulté, ont été inspirés à Aristide et à ses camarades ; parmi eux, on trouve un Crétois, nommé Évarestos :

23. [41] Ainsi Évarestos de Crète, I'un de ceux qui s'adonnent à la philosophie, venu d'Égypte pour s'enquêter sur ce qu'on disait du dieu - il était devenu mon familier ( $ү \vee \dot{\omega} \rho \mu \circ \varsigma$ ) et mon compagnon (бuví日ns) lors de mon séjour en Égypte - me dit que le dieu lui avait ordonné de m'encourager aux discours, car c'était là ce qui me convenait mieux que toute autre chose (traduction de A.-J. Festugière) [42].

On ne connaît rien de concret sur la pensée philosophique d'Évarestos, [43] Aristide étant le seul auteur antique à le mentionner, et l'unique conclusion que l'on peut tirer de l'extrait précédent est que le lien entre les deux hommes était garanti par leur prédisposition à croire. En outre, le passage en question constitue un nouveau témoignage des rencontres qu'Aristide a effectuées lors de son séjour égyptien : sans surprise, il était entouré d'hommes cultivés qui savaient apprécier son talent rhétorique.

Toutefois, Remus a fait sortir de l'oubli une proposition de Behr [44], qui tente d'établir un autre maillon, moins métaphysique, entre les deux compagnons par l'identification de l'ami crétois de l'orateur avec un philosophe de l'entourage du jurisconsulte P. Saluius Iulianus :

Q. Aelio / Egrilio / Euareto / philosopho / amico Salui / Iuliani, Aelia / Timoclia [ux]or / cum / filis. CIL XIII $8159=$ ILS 7776 (inter Bonnam et Coloniam).

Le célèbre juriste est devenu gouverneur de la Germanie Inférieure vers 161 et la stèle funéraire du philosophe grec doit dater de la même période. D'après le $\S 9$ de I'Or. 48, Iulianus se trouvait en 145 , à savoir peu avant les retrouvailles d'Aristide et d'Évarestos, dans le même sanctuaire d'Asclépios que notre sophiste :

\begin{abstract}
Or., 50, 9. [45] Mais pour l'heure, commençons par ce point entre autres : comment, lorsque je fus arrivé au sanctuaire, la première nuit le dieu apparut à mon nourricier sous l'aspect de Saluius, le présent consul. Qui est Saluius, je ne le savais pas encore; il se trouvait alors être assidu au temple auprès du dieu. Mon nourricier dit donc que, sous cet aspect, le dieu s'entretint avec lui sur mes discours, et qu'entre autres choses il les désigna sous les noms des « Discours sacrés » (trad. A.-J. Festugière).
\end{abstract}

Iulianus n'est pas désigné par son prénom ou par son cognomen, mais par son nomen, sans doute pour rappeler via l'étymologie l'attachement du Romain au

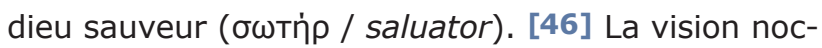
turne du nourricier d'Aristide occupe une place importante dans les innumérables rêves qui sont relatés dans les Or. 47-52, car c'est le songe qui suggère à Aristide la forme du ípòs $\lambda$ óyos pour ses textes relatifs à Asclépios. [47] L'apparition d'un fidèle, ignoré jusqu'alors par le sophiste, devient le messager et la preuve des pouvoirs du dieu, car une fois l'inconnu identifié à un fonctionnaire destiné à une brillante carrière politique, il pourrait se révéler d'une grande utilité pour le sophiste.

Pourtant, aussi séduisante que puisse paraître la suggestion de Behr, elle se heurte à un détail embarrassant : I'inscription latine et les manuscrits de l'Or. 50 ne conservent pas le même nom et comme les formes Eúápetos et Eưó́pєoTos sont attestées ailleurs, il n'est pas facile de rejeter l'une des deux, bien qu'Eúápeotos soit de loin le plus répandu des deux noms [48].

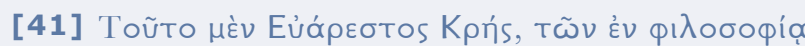

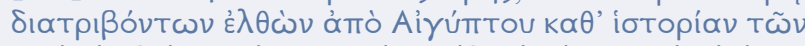

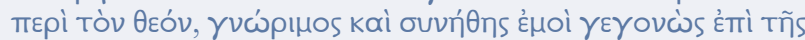

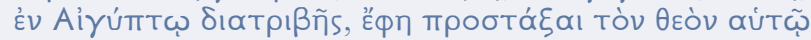

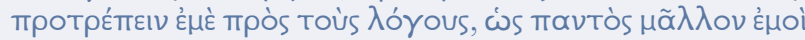
троó́KOVTOS.

[42] FESTUGiÈre 1986.

[43] BEHR 1981, p. 436, n. 39, I'associe sans hésitation et sans fondement à l'école platonicienne, mais PUECH 2000 a raison de ne pas accepter cette suggestion.

[44] Remus 1996, p. 150, 158 et 173, n. 48. La suggestion a initialement été présentée dans une note marginale de BEHR 1973, que H. Remus a pu consulter, avant qu'elle ne soit mieux élaborée dans BEHR 1994, p. 1155-1157.

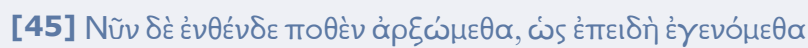

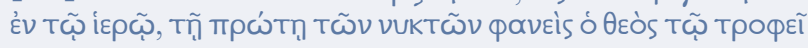

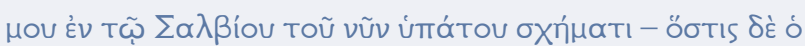

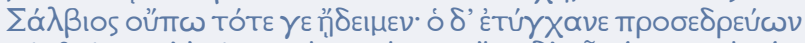

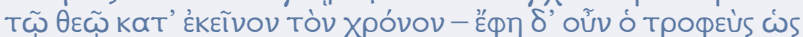

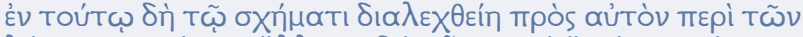

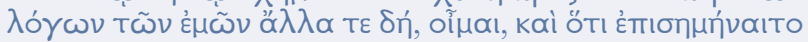

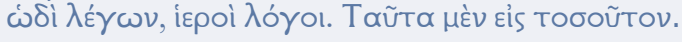

[46] BEHR 1981, p. 429, n. 18, et BEHR 1994, p. 1157-1158.

[47] Voir TASSEVA 2009 ; DOWNIE 2013, p. 47-48.

[48] Eúó́petos : c'est le nom d'un tragédien du IV $\mathrm{s}$. (IG $\mathrm{II}^{2}, 2319-2323$; TrGF 85) auquel s'ajoute une occurrence incertaine dans $I G \mathrm{~V}, 2,116$ (la terminaison -oc est ajoutée par les éditeurs modernes); le génitif Eủapétou semble provenir le plus souvent du nom Eủapétᄁs. Eưápeotos: présent dans 258 inscriptions de la base de données http:// epigraphy.packhum.org ; deux autres personnes nommées Eủópeotos sont mentionnées par Flavius Josèphe (Antiquités Juives, XIX, 145 et Guerre des Juifs, I, 532). 
W. Henzen [49], le premier éditeur de l'inscription, ne fait aucun effort pour associer Euaretus à l'ami crétois d'Aristide et se contente de noter que le philosophe devait être d'origine grecque et avoir obtenu la civitas Romana grâce à Q. Egrillius Plarianus [50]. Qui plus est, la $R E$, la $P I R$ [51] et, de façon encore plus résolue, le Dictionnaire des philosophes antiques [52] distinguent le Grec appartenant à l'entourage de Saluius d'avec le philosophe de I'Or. 50. Enfin, les historiens qui se penchent sur I'ILS 7776 ignorent aussi l'hypothèse de Behr [53].

Dans le texte de l'inscription, Euaretus se présente comme un supernomen qui vient s'ajouter aux tria nomina, sans pour autant être précédé de qui et, sive ou signo/signum à l'instar de la grande majorité d'agnomina et de signa répertoriés dans les inscriptions latines [54]. Mais Aristide a également ajouté le surnom $\Theta \varepsilon$ có $\omega \rho$ os à ses noms romains en guise de signe de dévotion à Asclépios [55] et dans une inscription gravée en son honneur [56], on voit son surnom pieux compléter ses tria nomina en se passant des formules introductives habituelles telles que ó

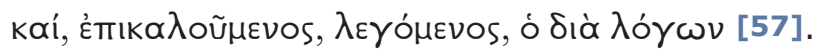

Bien qu'il soit risqué de dire avec certitude que le philosophe de la stèle et celui qui est mentionné dans I'Or. 50 sont la même personne, le rapprochement de Behr et de Remus ne peut être simplement négligé, vu la rareté de la forme Eưópetos dans les sources grecques et l'importance qu'Aristide donne aux noms acquis ou utilisés dans la communauté des adorateurs d'Asclépios. Quoi qu'il en soit, le texte de la stèle funéraire confirme que Iulianus fut une heureuse suggestion de la part du dieu, car il s'agissait d'un haut fonctionnaire romain souvent entouré d'intellectuels grecs.

Si I'on attribue la déformation du nom du comes de Iulianus à une faute du lapicide ou de celui qui a préparé le texte de la dédicace, et que l'on accepte que l'Euare<s>tus de l'inscription est l'ami d'Aristide, on se trouve devant une amitié gréco-romaine

[49] HeNZEN 1856, p. 125, no 5600.

[50] MERLIN 1942, p. 242, n. 2, l'identifie avec le proconsul en fonction entre 158 et 159 .

[51] ROHDEN 1894 ; PIR ${ }^{2}$ A 171.

[52] Voir PUECH 2000 : « n 185, Évarestos de Crète » et « $n^{\circ} 186$, Evaretus (Q. Aelius Egrilius) ».

[53] Voir, par exemple, le récent article de ECK 2012, p. $174-175$.

[54] KAJANTO 1966 , p. 5-8.

[55] Or., 50, § 53-55 et § 70.

[56] PUECH 2002, p. 140. Voir infra.

[57] Sur les formules introductives, voir CALDERINI 1941, triangulaire qui semble se construire en trois phases : deux Grecs, hommes de lettres et issus de contrées éloignées, se croisent d'abord en Égypte, peut-être à Alexandrie, et leur amitié est consolidée à Pergame par une quête spirituelle commune dans le cadre du culte d'Asclépios ; c'est à ce moment-là que se présente l'occasion de faire la connaissance d'un Romain appartenant aux hautes sphères du pouvoir. [58]

La stabilité des contacts avec les membres de l'administration romaine dans le réseau d'un sophiste assurait sa liberté oratoire tout autant que sa liberté de circulation et Aristide avait conscience de la manière dont ce double privilège pourrait lui être octroyé : à deux reprises, aux § 59-61 de son éloge de Rome (Or., 26) et au § 91 de l'Or. 36, il insiste sur la facilité de voyager dans l'empire grâce à la Pax Romana et à la qualité de citoyen romain. Aussi Aristide s'est-il tourné vers les autorités en 153 pour se faire exonérer de l'office d'irénarque. [59] Parmi les lettres de soutien qui lui ont été adressées (Or., 50, § 75), il met naturellement en vedette la plus décisive, une épître signée par les deux empereurs, Antonin le Pieux et Marc Aurèle, qui ont tranché en sa faveur. Par une heureuse coïncidence, ou, suivant l'orateur, grâce à la bienveillance d'Asclépios, d'autres lettres élogieuses et utiles lui sont parvenues :

Or., 50, 75. D’Héliodore, aussi, qui était devenu préfet d'Égypte, il m'arrive en même temps que la lettre impériale, deux lettres, I'une pour moi, l'autre à mon sujet, pour le proconsul, toutes pleines d'honneur et de brillants éloges ; elles avaient été écrites bien avant mes difficultés, mais me parvinrent alors juste à point (trad. de A.-J. Festugière) [60].

Le dignitaire romain en question est identifié à C. Avidius Heliodorus, père du futur usurpateur C. Avidius Cassius. Il occupait le poste du préfet d'Égypte entre 137 et 142 [61], c'est-à-dire à l'époque

p. 221-260. Le cas des supernomina sans formule introductive est discuté à la p. 248 ; entre les p. 249 et 253, l'auteure signale les emplois des pseudonymes à la place des prénoms. Cf. Broux 2014.

[58] BEHR 1994, p. 1157 suppose gratuitement que la rencontre entre Évarestos et Julianus a eu lieu en Égypte, car le juriste y a aussi résidé.

[59] Sur cette affaire, voir BRÉLAZ 2005, p. 95-96 et p. $108-110$.

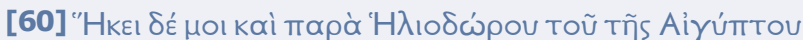

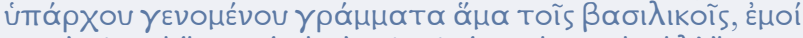

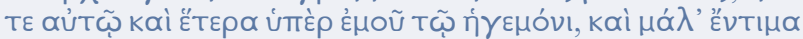

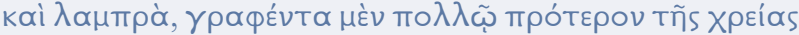

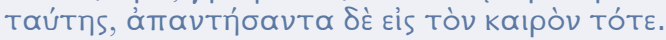


où Aristide séjournait au pays du Nil. L'Histoire Auguste (Hadr., 16, 10) mentionne le préfet parmi les philosophes de l'entourage de l'empereur Hadrien, tandis que selon Cassius Dion (LXIX, 3, 5), Héliodore a également assuré la fonction de procurator ab epistulis Graecis. Si Aristide met en évidence le mandat de préfet en Égypte parmi tous les offices occupés par Héliodore, le choix s'avère significatif des conditions dans lesquelles les deux hommes se sont rencontrés, d'autant plus que, quand le sophiste organisait son voyage en Égypte, il devait trouver un moyen pour circuler librement dans cette partie de l'Empire.

Une des fonctions principales du préfet était de contrôler l'accès en Égypte et tous les citoyens romains, y compris les voyageurs qui se déplaçaient d'Alexandrie vers la $\chi \tilde{\omega} \rho \alpha$ ou d'un nome à l'autre, devaient être munis des laissez-passer (ámóoto issus des services préfectoraux [62]. Aristide, lors de ses excursions en Haute-Égypte et en Éthiopie, semble disposer de documents de voyage, voire de lettres de recommandation, signés par une personne haut placée. Même s'il ne s'agissait pas d'Héliodore, le contact d'Aristide devait faire partie de l'entourage du préfet. L'importance que les autorités locales accordaient au jeune sophiste apparaît nettement dans un épisode décrit au § 49 de I'Or. 36. Après une visite décevante de la première Cataracte du Nil et malgré ses problèmes de santé [63], Aristide n'hésite pas à

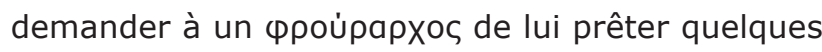
légionnaires pour y retourner et pour obliger les pêcheurs locaux à lui montrer le site ainsi qu'à lui faire leur fameuse démonstration nautique [64]. Le fait que le militaire, malgré ses réticences, cède devant I'opiniâtreté d'Aristide « en se comportant en fami-

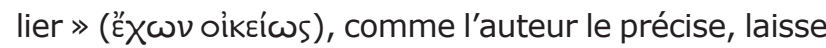
entendre qu'il était au courant des contacts dont son hôte disposait auprès de ses supérieurs.

Un autre élément qui corrobore I'hypothèse des appuis d'Aristide auprès de l'administration romaine de I'Égypte provient de la Lettre aux Rhodiens sur la concorde (Or., 24). Au § 3 Aristide souligne l'intérêt

[61] JÖRDENS 2009, p. 529.

[62] PURPURA 2002, p. 131-156.

[63] RoBIANO 2011, p. 146-147, évoque l'envie du sophiste « de mettre en valeur son propre courage », qui n'est pas sans rappeler la mise en scène « athlétique » de ses guérisons spectaculaires ; sur cela, voir DowNIE 2013, p. $115-117$.

[64] Décrite par Diodore de Sicile ( $I, 32,10)$, Sénèque (Questions Naturelles, Iva, 2,6) et Strabon (XVII, 1, 49). Cf. BeRnAND 1969, n 158, p. 127-138.

[65] Voir HEBERDEY 1897 et KoKKINIA 2000.

[66] BEHR 1968, p. 16, n. 48. qu'il a toujours porté à l'égard de la cité rhodienne en rappelant à ses destinataires que, quand le Dodécanèse et la Lycie ont été frappés par un séisme (entre 139 et 141) [65], il a rencontré et soutenu les ambassadeurs des sinistrés en Égypte. La nature de I'assistance offerte n'est pas énoncée : Behr [66] a suggéré qu'Aristide aurait prononcé à cette occasion un simple discours de consolation, mais sa conjecture a légitimement été contestée par Jones [67]. Il serait tentant de voir derrière ces propos de l'orateur une allusion à une aide plus concrète, comme un discours en faveur des demandes rhodiennes adressé aux autorités romaines. Encore une fois, l'amitié entre Aristide et un haut fonctionnaire cultivé de l'Empire semble être initialement fondée sur des intérêts intellectuels communs avant de profiter à l'otium cum litteris du sophiste et, peutêtre, aux besoins de ses compatriotes.

En fin de compte, le soutient rassurant d'Évarestos et d'Héliodore ainsi que le souvenir du secours apporté par Aristide aux Rhodiens plaident en faveur d'une activité rhétorique en Égypte. Bien qu'elle soit absente du corpus actuel, elle est confirmée par une source épigraphique :

La cité d'Alexandrie et Hermoupolis la Grande et le conseil des Antinoéens Nouveaux Grecs et les Grecs du Delta d'Égypte, comme ceux qui habitent le nome thébaïque ont honoré Publius Aelius Aristide Théodore, pour son excellence

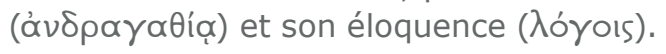

$$
\text { CIG III } 479=\text { OGIS } 709=\text { IGR IV } 1070 \text { [68] }
$$

L'inscription, découverte en 1743 en Vénétie, a suscité de vives discussions portant sur son authenticité et sur son contenu [69]. Gravée indubitablement après le voyage d'Aristide en Égypte et le début de sa

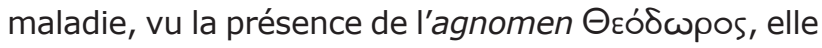
a initialement été interprétée comme un hommage collectif de l'hellénisme d'Égypte envers le sophiste [70]. Pourtant, J. Bingen a mis en doute sa provenance alexandrine, en privilégiant une origine smyrniote,

[67] JONES 1990, p. 515, n. 6.

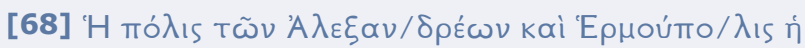

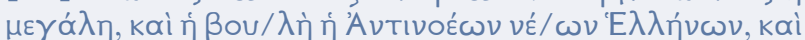

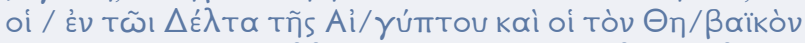

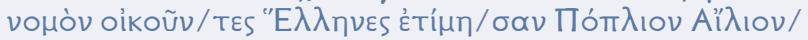

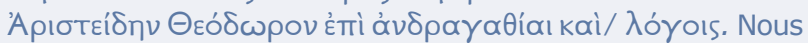
reprenons la traduction de PUECH 2002, p. 141, avec de petites modifications.

[69] L'historique de la découverte et les débats survenus sont exposés dans l'article de QUET 1992.

[70] BEHR 1968, p. 111, n. 64 ; BERNAND 1984, p. 66-72, n. 4 ter. 
jadis soupçonnée par Boulanger [71], et a cherché à dégager les тóno rhétoriques d'une terminologie administrative qui ne correspond pas aux réalités politiques de l'Égypte romaine : il s'agirait donc d'une « dédicace fictive » composée et érigée à Smyrne par des amis ou des disciples d'Aristide. Enfin, Bingen n'a pas hésité à associer le texte de l'inscription à un plus long décret honorifique de la base de la statue de bronze qui, selon les Vies des Sophistes (II, 582) de Philostrate, fut érigée par les Grecs, les Italiens et les Égyptiens dans la cité ionienne en I'honneur de I'orateur ; en partant de ces nouvelles données, Quet et Puech [72] se sont notamment penchées sur le mot "E $\mathrm{\lambda} \lambda \eta \eta v \varepsilon s$ par lequel on désignait parfois les disciples et les auditeurs des orateurs. Des élèves d'Aristide ont peut-être essayé de rappeler le voyage et les activités rhétoriques de leur maître en Égypte, en résumant de précédents décrets honorifiques issus du pays du Nil, sans pour autant imiter fidèlement leur contenu ni usurper le pouvoir des entités politiques d'une autre province.

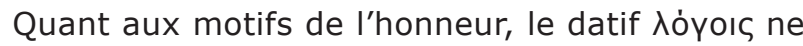
surprend pas, puisqu'il est question d'un sophiste,

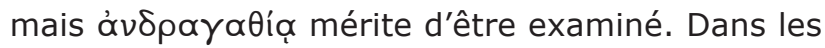
inscriptions des époques classique et hellénistique, le terme fait référence à des services rendus, le plus souvent de nature martiale [73], mais aussi de caractère civique ou diplomatique [74]. Pourtant, à

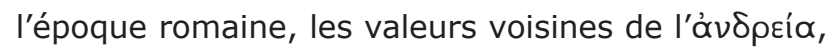

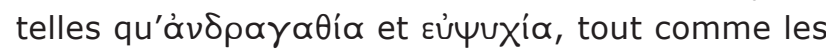

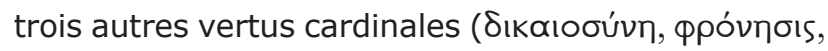

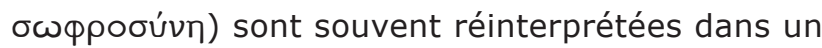
contexte culturel et agonistique [75].

Dans le corpus des inscriptions grecques de l'ère impériale, les distinctions honorifiques d'Aelius Sospes [76] présentent des similitudes avec celles d'Aristide. Sospes était un orateur issu d'une lignée de notables romano-corinthiens qui a également eu le plaisir de voir ses concitoyens lui ériger une statue

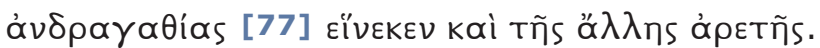

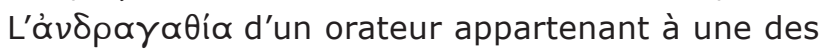
plus puissantes familles du Péloponnèse ne devait pas être abstraite, mais fondée sur des actions concrètes,

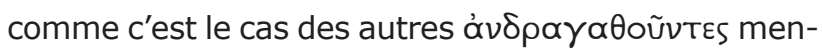
tionnés par les inscriptions des époques précédentes. Mais pour quelles raisons précises Aristide a-t-il pu être honoré de la même manière ? Vu son caractère [78] et sa santé fragile, le sophiste ne devait pas avoir I'habitude de s'impliquer dans des concours rhétoriques en tant que compétiteur ou organisateur. Toutefois, grâce aux Or. 23, 24 et 27 , on sait que les querelles entre cités ne laissaient pas indifférent Aristide qui prônait la concorde et la solidarité entre Hellènes. S'il subsistait une trace d'un discours portant sur les différends politiques en Égypte, une nouvelle expression de son envie de soutenir ses compatriotes expliquerait aisément les honneurs rendus par les instances civiques de la province. Pourtant, seuls les $\S 33$ et 34 de l'Or. 36 permettent d'apercevoir furtivement Aristide esquisser des problèmes de I'administration romaine dans cette province et être concerné par les ennuis des sujets de l'Empire :
33 [79] Quand je naviguais en Égypte pour la deuxième fois, en remontant le cours du Nil vers le sommet, nous rencontrâmes, près de la région de Thèbes, dans un nome appelé Hermounthis, un exilé de la cité basse, du nom de Draukos ;

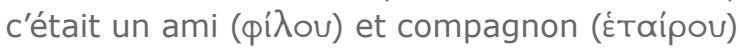 qui, I'ayant vu là-bas, le mit en relation (бuбтn்- бavTOS) avec nous. Quand il advint que son exil fut levé et qu'il revint près de la mer, il venait souvent nous voir ( $\pi \rho \circ \sigma n ূ \varepsilon l)$ et nous le traitions,

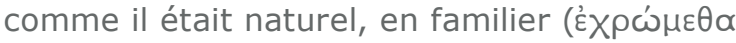 oikzí(s).

[71] BINGEN 1987. BouLANGER 1923, p. 131, voyait derrière cette dédicace une association égyptienne à Smyrne qui souhaitait honorer l'orateur pour son aide à la reconstruction de la cité après le séisme de 177-178.

[72] QUET 1992, p. 396-401 ; PUECH 2002, p. 143-144. [73] Voir à titre indicatif : IG $\mathrm{I}^{3}, 125$ (405/404 av. J.-C.) IG II ${ }^{2}, 145$ (403/402 av. J.-C.) ; IG II 2,276 (336/335 av. J.-C.) ; IG II², 500 (302/301 av. J.-C.)

[74] IG II², 1138 (403/402 av. J.-C., chorégie) ; IG II² 23 (398/397 av. J.-C., proxénie et évergétisme) ; IG II²,

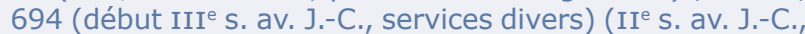
philathénaïsme) ; IG II ${ }^{2}, 1261$ (fin IV ${ }^{e}$ s., contribution à I'organisation du cortège d'Adonis).

[75] LAFOND 2006, p. 38-42.

[76] KENT, Corinth VIII, 3, 226 = KAPETANOPOULOS, Mnemosyne 22, 1969, p. 80-82 = PUECH 2002, n 241, p. 452-453. Son grand-père, Antonius Sospes, s'était illustré comme agonothète aux Jeux Isthmiques, responsable des sacrifices et évergète à Corinthe ainsi que comme orateur à Athènes (voir Plutarque, Propos de Table, VIII, 4 et IX, 5, 12 et 13 ; Philostrate, Vies des sophistes, II, 591).

[77] Notons que, cette fois, PUECH 2002, p. 452, préfère

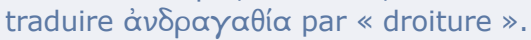

[78] Philostrate (Vies des sophistes, II, 581-582) écrit qu'Aristide évitait les improvisations rhétoriques et supportait mal l'absence de louanges.

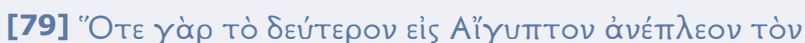

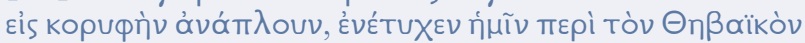

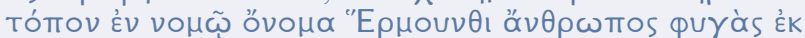

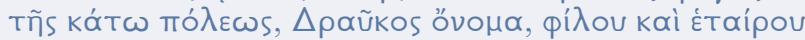

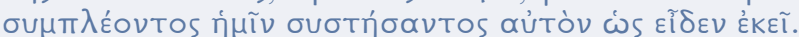

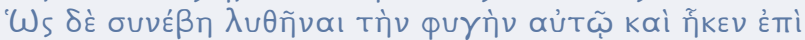

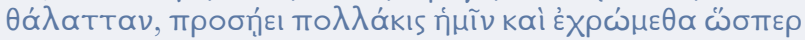

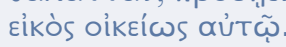


L'entrée de l'individu dans le réseau égyptien d'Aristide serait d'emblée jugée banale et fortuite, si Draukos n'était pas condamné par les autorités romaines et s'il ne se liait pas d'amitié avec des personnes qui, comme il a été montré, sont en bons termes avec I'entourage d'Héliodore. Le préfet d'Égypte, doté de pouvoirs juridiques étendus, était le juge suprême de la province et le responsable pour l'exécution de toute peine relative à l'exil ou à la damnatio ad metalla [80]. La suite du texte montre que Draukos ne pouvait être un simple criminel, car on le voit participer aux promenades et aux discussions d'un groupe d'érudits. De plus, Aristide n'hésite pas à utiliser son témoignage pour réfuter la plus populaire théorie sur la crue du Nil, celle du Péripatos [81] :

34 C'est ainsi qu'un jour, vers le soir, nous nous promenions simplement dans la grande rue qui longe les portiques, tandis que les vents étésiens étaient forts. Nous tournâmes, donc, nos yeux vers les nuages et quelqu'un parmi les présents dit : « Voici, je suppose, les souffles d'air du Nil » - en effet, il les appela à peu près de cette façon. Et Draukos se mit à rire. Et quand je lui demandai ce qu'il entendait par là, il répondit : « Ne sais-tu pas que j'ai gaspillé trois ans consécutifs à l'extrémité de l'Égypte ? » « Je le sais », dis-je, «mais que veux-tu dire ? » « Je veux dire que moi, qui ai passé tant de temps en observant partout, je n'ai pas pu voir de nuage à cet endroit en été ; au contraire, j'ai vu l'atmosphère rester immobile, comme les peintures. Eh bien donc », dit-il, « je crois voir ce qui est possible de se produire; pourtant je n'ai pas vu de nuage, ces nuages, qui, à votre avis, », dit-il, « sont poussés vers la Haute-Égypte et, en crevant au Sud, remplissent le Nil [82] ».

Draukos, en entendant la communis opinio de la bouche d'un des promeneurs, démasque en riant un mensonge accepté par ceux qui n'ont pas vécu l'expérience de la déportation dans le désert égyptien. L'insistance de l'ancien $\varphi$ uyác sur sa peine purgée n'est pas sans rappeler un топо́ présent chez une pléiade de philosophes et d'orateurs, notamment dans des consolations (napnyopiaı) et des éloges paradoxaux : «le bon usage de l'exil ». De

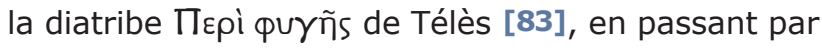
Sénèque (Ad Helviam), Mussonius Rufus [84] (fr. IX :

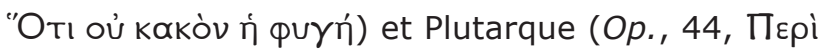

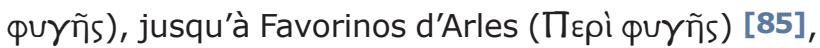

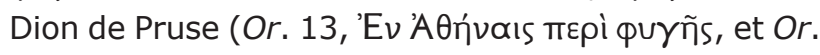

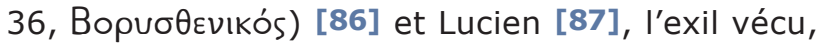
observé ou imaginé a nombre de conséquences bénignes : au lieu de détruire moralement les condamnés, il leur permet d'enrichir leurs connaissances, de se débarrasser des biens inutiles, d'éviter l'agitation de la ville, de se défaire des idées fausses, de devenir de vrais cosmopolites et de modifier radicalement leur angle de vue sur leur patrie et sur le pouvoir exercé par les grands. T. Whitmarsh [88] note sommairement que l'expérience de la $\varphi$ yyn fait souvent partie de la stratégie littéraire des représentants de la Seconde Sophistique, puisqu'elle est considérée comme un moyen d'affirmation de leur grécité et de leur nappnoía vis-à-vis du pouvoir.

Les fréquentations et le comportement de Draukos nous permettent de le joindre aux cercles intellectuels alexandrins et il serait séduisant de chercher la cause de sa relegatio dans une activité rhétorique, politique ou philosophique plutôt gênante. Son cas pourrait faire penser aux esprits subversifs des Acta Alexandrinorum qui par leurs actes ou par leurs propos défiaient les autorités romaines : A. Harker, dans la plus récente synthèse portant sur le corpus des Acta [89], n’hésite pas à mettre en parallèle l'idéologie hellénocentrique des sophistes, tels qu'Aristide [90] et Dion de Pruse, avec la fierté patriotique des martyrs alexandrins,
[80] BASTIANINI 1988, p. 351-356 ; MATTER 1999. [81] Nous nous permettons de renvoyer à RAÏOs 2013.

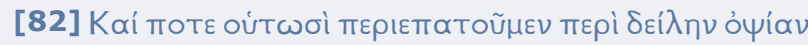

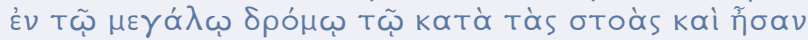

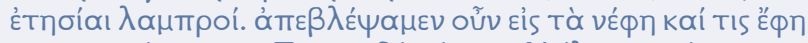

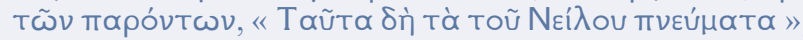

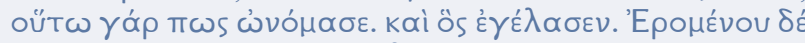

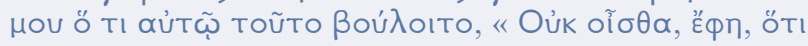

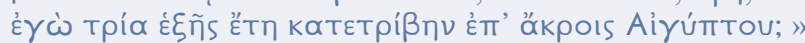

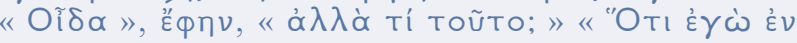

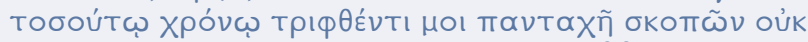

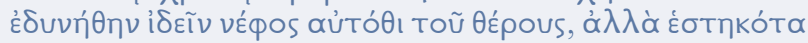

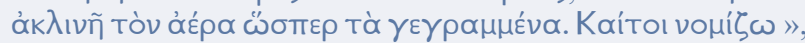

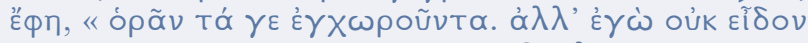

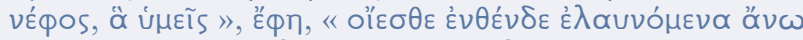

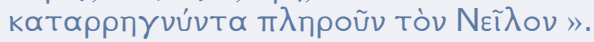

[83] Voir Fuentes GonzÁLez 1998, p. 274-362 (fragment III).

[84] Exilé au moins une fois à Gyaros sous Néron. Voir Goulet-CAzé 2005.

[85] Pour une récente présentation globale du $\Pi \varepsilon \rho i$ фuY voir AMATO \& MARGANNE 2015.

[86] Voir la notice de BOST-POUDERON 2011, p. 105-137.

[87] Op., 27, 9 (I'errance comme une conditio sine qua non du cynisme) et 59, 41 (les bons historiens sont des apatrides).

[88] WHITMARSH 2001.

[89] HARKER 2008, p. 148-149 et p. 167-173.

[90] Notons qu'Aristide (Or., 3, § 671), tout comme les « martyrs païens », semble tenir en piètre estime les penseurs judéo-chrétiens. 
qui comptaient dans leurs rangs nombre d'hommes cultivés.

L'hypothèse que nous avançons, loin d'être à l'abri des objections sur la base de nouvelles découvertes, est en accord avec les remarques de L. Pernot portant sur les relations qu'Aristide entretenait avec les autorités romaines [91]: le sophiste ne s'est jamais opposé ouvertement à ses maîtres et n'a jamais subi des peines graves, tout en montrant sa préférence pour un détachement progressif du pouvoir afin de protéger sa vie contemplative et ses convictions spirituelles. Comme Aristide maîtrisait l'oratio figurata, le double discours, autant que la rhétorique de l'éloge, on ne s'étonne pas face à l'apparition furtive d'un homme condamné par la même instance qui soutient le sophiste ou à la description des conditions de vie lamentables des damnati ad metalla (Or., 36, $\S 67)$, qui contraste avec l'image paisible de l'Égypte pacifiée [92].

\section{CONCLUSIONS}

Notre étude d'une fraction du réseau d'Aristide montre qu'examiner les liens tissés entre le sophiste, son public et son entourage par l'intermédiaire de sa propre œuvre et des témoignages extérieurs (biographies et documents épigraphiques ou papyrologiques) résume convenablement sa vie et sa carrière. D'un côté, la thématique de I'Or. 36 dévoile un public cultivé qui s'intéresse à des questions scientifiques ou spirituelles et avec lequel I'orateur aime interagir ; puis, les récits relatifs au séjour égyptien alimentent I'amour-propre d'Aristide et mettent en relief sa conception de la прóvoı divine qui est censée actionner sa vie et lui assurer une carrière rhétorique réussie. De l'autre côté, si le lecteur se concentre sur les personnes, il voit défiler sous ses yeux des savants confirmés, des prêtres vénérables, de puissants officiers et quelques esprits réfractaires, tels que Draukos. Tous ces individus permettent de dégager les buts divers qu'Aristide veut atteindre par son œuvre : faire preuve de sa grande culture et se faire reconnaître comme un orateur polyvalent, souligner sa protection par des Romains philhellènes, sans pour autant perdre sa liberté ou son intégrité, obéir aux dieux, aider les autres Grecs et être en retour honoré par eux.

[91] PERNOT 2008

[92] POLAŃSKI 2006, p. 247-248.

\section{BIBLIOGRAPHIE}

Amato, Eugenio \& Marganne, Marie-Hélène (éd.), 2015, Le traité sur l'exil de Favorinos d'Arles. Papyrologie, philologie et littérature, Rennes.

Bastianini, Guido, 1988, « Un ordine di scarcerazione: P. Berol. 8997 (ChLA X 421) », dans Basil G. Mandilaras (éd.), Proceedings of the XVIII International Congress of Papyrology, Athens, 25-31 May 1986, Athinai, p. 351-356.

BeHR, Charles A., 1968, Aristides and the Sacred Tales, Amsterdam.

Berr, Charles A., 1973, Aristides. I: Panathenaic Oration and in Defense of Oratory, Text and Translation, Cambridge, Mass. (The Loeb classical library 458).

Behr, Charles A., 1981, P. Aelius Aristides. The Complete Works Translated into English. II: Orations XVII-LIII, Leiden. BerR, Charles A., 1994, "Studies on the Biography of Aelius Aristides », Aufstieg und Niedergang der römischen Welt II, 34, 2, Berlin - New York, p. 1140-1233.

Bernand, Étienne, 1969, Les inscriptions grecques et latines de Philae. II : Haut et Bas Empire, Paris.

Bernand, André, 1984, Les portes du désert. Recueil d'inscriptions grecques d'Antinooupolis, Tentyris, Koptos, Apollonopolis Parva et Apollonopolis Magna, Paris.

Bingen, Jean, 1987, « Aelius Aristide, OGIS 709 et les "Grecs d'Égypte" », dans Jean Servais, Tony Hackens \& Brigitte Servais-Soyez (éd.), Stemmata. Mélanges de philologie, d'histoire et d'archéologie grecques offerts à Jules Labarbe, Liège - Louvain-la-Neuve, p. 173-185.

Bonneau, Danielle, 1964, La crue du Nil, divinité égyptienne à travers mille ans d'histoire (332 av. J.-C. - 641 ap. J.-C.), Paris. 
Bost-Pouderon, Cécile, 2011, Dion de Pruse dit Dion Chrysostome, Euvres. Premier discours à Tarse (Or. XXXIII), Second discours à Tarse (Or. XXXIV), Discours à Célènes de Phrygie (Or. XXXV), Discours Borysthénitique (Or. XXXVI), Paris (Collection des universités de France. Série grecque 480).

Boulanger, André, 1923, Aelius Aristide et la sophistique dans la province d'Asie au II siècle de notre ère, Paris.

BOWERSOCK, Glen, 1969, Greek sophists in the Roman Empire, Oxford.

BRÉLAZ, Cédric, 2005, La sécurité publique en Asie Mineure sous le Principat (I ${ }^{e r-I I I}{ }^{e}$ s. ap. J.-C.). Institutions municipales et institutions impériales dans l'Orient romain, Basel.

BrouX, Yanne, 2014, Double Names in Roman Egypt: A Prosopography [en ligne : www.trismegistos.org/downloads/TOP_8]. CalderinI, Rita, 1941, « Richerche sul doppio nome personale nell'Egitto greco-romano », Aegyptus 21, p. 221-260.

DownIE, Janet, 2013, At the Limits of Art. A Literary Study of Aelius Aristides' Hieroi Logoi, Oxford.

ECK, Werner, 2012, «Senatorisches Leben jenseits von Politik, Militär und Administration: Die öffentliche Repräsentation der intellektuellen Seite der Führungsschicht », dans Marcel van Ackeren \& Jan Opsomer (éd.), Selbstbetrachtungen und Selbstdarstellungen der Philosoph und Kaiser Marc Aurel im interdisziplinären Licht, Köln, p. 169-186.

Edelstein, Emma \& Edelstein, Ludwig, 1945, Asclepius. A Collection and Interpretation of the Testimonies, 2 vol., Baltimore.

Festugrère, André-Jean, 1986, Aelius Aristide. Discours Sacrés : rêve, religion, médecine au II siècle après J. -C., Paris. FoertMEYer, Victoria Ann, 1989, Tourism in Graeco-Roman Egypt, Princeton University (thèse inédite).

Fournel, Eugénie, 2015, «La foi d'Aristide à l'épreuve de la maladie : Confiance en soi dans les discours sacrés », Camenulae 12, p. 1-13.

Fuentes GonzÁlez, Pedro Pablo, 1988, Les diatribes de Télès, Paris.

Goeken, Johann, 2012, Aelius Aristide et la rhétorique de l'hymne en prose, Turnhout.

Goulet-CAzÉ, Marie-Odile, 2005, s. v. Mussonius Rufus (C.), dans Richard Goulet (éd.), Dictionnaire des philosophes antiques IV, Paris, p. 555-572.

HARKER, Andrew, 2008, Loyalty and Dissidence in Roman Egypt. The case of Acta Alexandrinorum, Cambridge.

HeberdeY, Rudolf, 1897, Opramoas. Inschriften vom Heroon zu Rhodiapolis, Wien.

Henzen, Wilhelm, 1856, Volumen tertium Collectionis Orellianae supplementa emendationesque exhibens, Zürich.

Hostern, Antony \& LaLANne, Sophie (éd.), 2012, Les voyages des empereurs dans l'Orient romain. Époques antonine et sévérienne, Arles.

JONES, Christopher P., 1990, « The Rhodian Oration Ascribed to Aelius Aristides », Classical Quarterly 40. 2, p. 514-522. JöRDENS, Andrea, 2009, Statthalterliche Verwaltung in der römischen Kaiserzeit. Studien zum praefectus Aegypti, Stuttgart. KAJANTO, Iiro, 1966, Supernomina. A Study in Latin Epigraphy, Helsinki.

KAYSER, Carl Ludwig, 1870-1871, Flavii Philostrati Opera auctiora, 2 vol., Leipzig (Bibliotheca scriptorum Graecorum et Romanorum Teubneriana).

KoKkInIA, Christina, 2000, Die Opramoas-Inschrift von Rhodiapolis. Euergetismus und soziale Elite in Lykien, Bonn.

LAFOND, Yves, 2006, La mémoire des cités dans le Péloponnèse d'époque romaine (II siècle avant J.-C.- III ${ }^{e}$ siècle après J.-C.), Rennes.

MANOLARAKI, Eleni, 2012, Noscendi Nilum cupido. Imagining Egypt from Lucan to Philostratus, Berlin.

MATter, Michel, 1999, «Privation de liberté et lieux de détention en Égypte romaine », dans Cécile Bertrand-Dagenbach, Alain Chauvot, Michel Matter \& Jean-Marie Salamito (éd.), Carcer I. Prison et privation de liberté dans I'antiquité classique, Paris, p. 98-113.

MerLIN, Alfred, 1942, «Un proconsul d'Afrique méconnu, Egrilius Plarianus », Comptes rendus des séances de l'Académie des Inscriptions et Belles-Lettres 86, p. 235-242

Parmeggiani, Giovanni, 2011, Eforo di Cuma. Studi di storiografia greca, Bologna.

Pernot, Laurent, 1989, s. v. Aristide (P. Aelius), dans Richard Goulet (dir.), Dictionnaire des philosophes antiques I, Paris, p. 358-366.

Pernot, Laurent, 1997, Éloges grecs de Rome, Paris.

Pernot, Laurent, 2002, « Les Discours Sacrés d'Aelius Aristide entre médecine, religion et rhétorique », Atti della Accademia Pontaniana 51, p. 369-383.

Pernot, Laurent, 2007, « Le livre grec au II è siècle ap. J.-C. d'après I'œuvre d'Aelius Aristide », Comptes rendus des séances de l'Académie des Inscriptions et Belles-Lettres 151, p. 933-965.

Pernot, Laurent, 2008, « Aelius Aristides and Rome», dans William V. Harris \& Brooke Holmes (éd.), Aelius Aristides between Greece, Rome and the Gods, Leiden - Boston, p. 175-203.

PolaŃSKI, Tomasz, 2005, « Boukoloi banditry: Greek perspectives on native resistance », Grazer Beiträge. Zeitschrift für die klassische Altertumswissenschaft 25, p. 229-248.

PostL, Brigitte, 1970, Die Bedeutung des Nil in der römischen Literatur mit besonderer Berücksichtigung der wichtigsten griechischen Autoren, Wien.

Puech, Bernadette, 2000, s. v. Évarestos de Crète, dans Richard Goulet (éd.), Dictionnaire des philosophes antiques III, Paris, p. 402.

PueCH, Bernadette, 2002, Orateurs et sophistes grecs dans les inscriptions d'époque impériale, Paris.

PuRPuRA, Gianfranco, 2002, « Passaporti romani », Aegyptus 82, p. 131-156.

QuATtRocelli, Luana, 2009, « Il pubblico dei Discorsi sacri di Elio Aristide », dans Giancarlo Abbamonte, Lorenzo Miletti \& Luigi Spina (éd.), Discorsi alla prova. Atti del quinto colloquio italo-francese: Discorsi pronunciati, discorsi ascoltati: contesti di eloquenza tra Grecia, Roma ed Europa, Napoli, p. 259-278.

QUET, Marie-Henriette, 1992, «L'inscription de Vérone en I'honneur d'Aelius Aristide et le rayonnement de la Seconde Sophistique chez les "Grecs d'Égypte" », Revue des Études Anciennes 94, p. 379-391. 
RAÏos, Constantin, 2011, Le «Discours égyptien » d'Aelius Aristide. Édition critique, traduction et commentaire, Université de Strasbourg (thèse inédite).

RAïos, Constantin, 2013, « Le Discours égyptien d'Aelius Aristide et l'autorité scientifique d'Aristote : accords et désaccords », dans Yves Lehmann (éd.), Aristoteles Romanus. La réception de la science aristotélicienne dans l'Empire gréco-romain, Turnhout, p. 513-524.

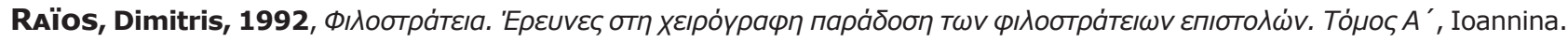
REARDON, Bryan P., 1971, Courants littéraires grecs des $I I^{e}$ et $I I I^{e}$ siècles ap. J.-C., Paris.

Remus, Harold, 1996, «Voluntary Associations and Networks. Aelius Aristides at the Asclepieion at Pergamum », dans John S. Klopenborg \& Stephen G. Wilsons (éd.), Voluntary associations in the Graeco-Roman World, London - New York, p. $146-175$.

ROBERT, Fabrice, 2013, « Aristide exégète, commentateur et critique dans le Discours égyptien », Schedae 2, p. 1-14. Robiano, Patrick, 2011, «Entre réalité et fiction, la frontière égypto-éthiopienne chez Aelius Aristide, Xénophon d'Éphèse, Philostrate et Héliodore d'Émèse », Kentron 27, p. 131-150.

Rohden, Paul von, 1894, s. v. Euaretus, RE I, 1, col. 492.

SAUNeron, Serge, 1988, Les prêtres de l'ancienne Égypte, Paris.

STERN, Menahem, 1980, Greek and Latin Authors on Jews and Judaism II, Jérusalem.

SYKUTRIS, Johannes, 1931, « Epistolographie », RE Supplement V, col. 204-205.

TASSEVA, Mina, 2009, «Le "discours sacré" (hieros logos) : une forme de rhétorique religieuse dans I'Antiquité grecque », dans Laurent Pernot (éd.), New Chapters in the History of Rhetoric, Leiden - Boston, p. 445-465.

VIX, Jean-Luc, 2010, L'enseignement de la rhétorique au II siècle ap. J.-C. à travers les discours 30-34 d'Aelius Aristide, Turnhout.

WhItMARSH, Tim, 2001, "Greece is the world": exile and identity in the Second Sophistic », dans Simon Goldhill (éd.), Being Greek under Rome. Cultural Identity, Second Sophistic and the Development of Empire, Cambridge, p. 269-305.

WhItMARSH, Tim, 2005, The Second Sophistic, Oxford.

Wilamowitz-Moellendorff, Ulrich von, 1925, «Der Rhetor Aristeides », Sitzungsberichte der Preussischen Akademie der Wissenschaften 28, p. 333-353. 